\section{Revisiting induction chemotherapy before radiotherapy for head and neck cancer, part l: carcinoma of non-nasopharyngeal sites}

\author{
"Future novel approaches will likely feature radiographic, proteomic \\ and genomic biomarkers used to define prognostic groups and guide \\ treatment selection with greater precision. Because of its flexibility and \\ adaptability, induction will likely continue to be a 'testing site' for new \\ chemotherapeutic, targeted or immunologic agents."
}

Christopher H Chapman', Upendra Parvathaneni² \& Sue S Yom ${ }^{*, 1}$

First draft submitted: 15 November 2016; Accepted for publication: 4 January 2017; Published online: 7 February 2017 and nasopharyngeal carcinomas, due to the unique history and biology of these cancer types. This first review will consider squamous cell carcinomas of the oral cavity, oropharynx, larynx and hypopharynx. Nasopharyngeal carcinoma will be addressed separately [1].

Early trials combining chemotherapy with radiation found encouraging response rates with cisplatin, especially when it was combined with 5-fluorouracil (5FU). By the 1990s, induction chemotherapy with cisplatin/5FU was the most common method of treating advanced head and neck cancer, although most of the evidence came from small, underpowered studies. This problem was addressed by meta-analyses, including the meta-analysis of chemotherapy in head and neck cancer (MACH-NC), which eventually combined 93 trials including over 17,000 patients utilizing individual patient data $[2,3]$. The MACH-NC study group found significantly improved overall survival from the use of concurrent chemotherapy as compared with radiation alone, but no

\section{KEYWORDS}

- antineoplastic agents

- antineoplastic combined chemotherapy protocols

- carcinoma • squamous cell

- chemotherapy • clinical trials

- combined modality therapy $\bullet$ head and neck neoplasms $\bullet$ induction therapy $\bullet$ molecular-targeted therapy $\bullet$ neoadjuvant therapy - sequential therapy • treatment outcomes

\section{“Delivering chemotherapy before radiation ... became a particularly attractive strategy, as full-dose chemotherapy administration was more tolerable when given as induction..."}


“Enthusiasm for induction chemotherapy resurged with the availability of a novel class of drugs, the taxanes." advantage was seen from induction chemotherapy. Intriguingly, however, while concurrent chemotherapy reduced distant failure (hazard ratio: 0.88 ), induction chemotherapy reduced distant failure further (hazard ratio: 0.73), although the difference was not significantly different from the rates seen with concurrent chemotherapy $(\mathrm{p}=0.12)$. This meta-analysis also found that platinum-containing chemotherapy regimens produced the greatest advantage in overall survival. This evidence, along with a few notable, adequately powered multi-institutional trials [4,5], established radiation with concurrent platinum-based chemotherapy by the mid-2000s as the standard of care for locally advanced head and neck cancer. Nonetheless, questions remained about the possibility of better distant control with induction chemotherapy.

\section{Induction as a strategy for organ preservation}

Induction was also investigated as a strategy for organ preservation in sites that would otherwise be managed surgically. This approach was most readily applicable to advanced laryngeal cancers, which can be successfully treated with laryngectomy, but at the cost of a reduced quality of life from losing one's natural voice. Published in 1991, the landmark Veterans Administration Larynx Trial compared upfront surgery followed by postoperative radiation versus induction cisplatin $/ 5 \mathrm{FU}$ followed by definitive-intent radiation for responders and surgery for nonresponders [6]. This strategy was able to achieve laryngeal sparing in nearly two-thirds of patients without compromising overall survival. Similar outcomes were seen in the European Organization for Research and Treatment of Cancer (EORTC) 24891 trial using induction cisplatin for hypopharyngeal cancers $[7,8]$.

Still, the ideal timing of chemotherapy was unclear. The Radiation Therapy Oncology Group (RTOG) 91-11 study attempted to address this by randomizing patients with advanced larynx cancer to induction cisplatin $/ 5 \mathrm{FU}$ followed by radiation, concurrent chemoradiotherapy with cisplatin, or radiation alone [9]. The long-term outcomes demonstrated improved rates of laryngeal preservation with concurrent chemotherapy $(82 \%)$ versus induction $(68 \%)$ or radiation alone (64\%) [10]. However, the primary composite end point of 'laryngectomy-free survival' showed no significant difference between the induction and concurrent arms ( 29 vs $24 \%$, respectively). This discrepancy was driven by an unexplained trend toward a higher 10-year death rate with concurrent chemotherapy ( 72 vs $61 \% ; \mathrm{p}=0.08$ ). There was no evidence of greater treatment-related late toxicity with concurrent chemotherapy, and only deaths 'unrelated to cancer or treatment' were significantly higher in the concurrent arm by post hoc analysis $(\mathrm{p}=0.03)$. Although possibly a chance occurrence, and while acknowledging the limited power of unplanned analyses conducted at long-term follow-up, this finding raises the question of whether unrecognized sequelae of concurrent chemoradiation increased late toxicities and deaths. While RTOG 91-11 generally showed concurrent chemotherapy to be superior in terms of the goal of laryngeal preservation, induction chemotherapy in larynx cancer has remained a defensible option due to this uncertainty.

\section{The emergence of taxane-based induction}

Enthusiasm for induction chemotherapy resurged with the availability of a novel class of drugs, the taxanes. These complex molecules inhibit microtubule depolymerization, interfering with mitosis. The first-in-class taxane, paclitaxel, was isolated from the Pacific yew tree in the 1960s. Due to early difficulties with production, clinical trials in head and neck cancer could not take place until the 1990s [11]. In 2005, Hitt et al. published a trial treating patients with three cycles of cisplatin/5FU before cisplatin chemoradiotherapy, and randomizing for the addition of paclitaxel [12]. This trial showed a significant improvement of overall survival with paclitaxel/cisplatin/5FU (TPF), which also resulted in less acute toxicity due to reduced 5FU dose in that arm. In 2007, the same issue of the New England Journal of Medicine featured the EORTC 24971/TAX 323 and TAX 324 trials $[13,14]$. Both trials randomly assigned for the addition of the taxane docetaxel to induction cisplatin $/ 5 \mathrm{FU}$, before either radiation alone (TAX 323) or before chemoradiotherapy using carboplatin (TAX 324). Both trials showed significant improvements in progression-free and overall survival rates with the addition of docetaxel.

The GORTEC 2000-01 study used a similar design for advanced larynx and hypopharynx cancers [15,16]. Patients were randomized to cisplatin/5FU induction with or without docetaxel, before definitive radiation with optional concurrent chemotherapy. Larynx preservation at 10 years was significantly improved from $47 \%$ to $70 \%$, but there were no differences in 
disease-free or overall survival rates. These four Phase III trials convincingly showed that adding a taxane could improve response and potentially improve survival compared to induction with cisplatin/5FU. However, none of these trials directly compared induction chemotherapy to the by-then-accepted standard of concurrent cisplatin-based chemoradiation alone. While two of the trials featured concurrent chemoradiation after induction (Hitt et al. and TAX 324) [12,14], $12 \%$ and $21 \%$ of patients, respectively, did not proceed from induction to radiation due to disease progression or toxicity from induction. Thus, while these studies established the TPF regimen as a new standard for induction, it remained unclear as to whether the benefits would outweigh the potential harm caused by reduced compliance to radiotherapy.

\section{Induction as a strategy to reduce distant metastases}

Supporting continued investigation were observations of a 'failure shift', with regimens that included induction chemotherapy resulting in higher rates of locoregional failure, versus the higher distant metastasis rates seen with concurrent-only chemoradiotherapy. This was especially seen in patients with advanced nodal disease [17]. Arguments were made that correct exploitation of induction chemotherapy might reduce distant failures (as in the MACH-NC meta-analysis) and thus increase survival. Two Phase III trials published in 2014 tested this hypothesis. The Spanish Head and Neck Cancer Group randomized patients to induction with TPF, induction with cisplatin/5FU (PF; before taxane induction results were known), or no induction before cisplatin chemoradiation [18]. Powered for progression-free survival, this trial found no significant advantage to induction versus chemoradiation by intention-to-treat analysis. Disturbingly, and in keeping with prior studies, about $30 \%$ of the induction patients did not proceed to chemoradiation, primarily due to hematologic toxicity. In the per-protocol analysis, induction chemotherapy improved the median progression-free survival by 5.2 months, but this only represented $81 \%$ of the randomized patients.

In the DeCIDE trial, patients were randomized to induction TPF before chemoradiation with docetaxel, 5FU and hydroxyurea [19]. To maximize the potential effects on distant failure, this trial was limited to patients with nodal stage N2a or greater. However, similar to the Spanish trial, DeCIDE found no differences in overall survival, although the study was underpowered for this primary end point due to slow accrual and lower-than-expected event rates. However, in this high-risk group, induction chemotherapy did significantly lower the rate of distant metastasis as first failure. There was also a trend toward improved overall survival with induction, in the higher risk subset of nodal stage N2c or greater. However, rates of hematologic toxicity in the induction arm again limited compliance with concurrent treatment, and thus the study was negative for survival differences.

The PARADIGM trial published in 2013 had a slightly different design [20]. Control arm patients received concurrent chemoradiation with cisplatin. The experimental arm started with three cycles of TPF induction, then radiation with weekly carboplatin (thought to be typically better tolerated than cisplatin) if the patient responded to induction, or weekly docetaxel with accelerated radiation if there was no response. Ideally, this design was aimed at shifting treatment intensity (and toxicity) to the higher risk patients, while improving compliance rates among lower risk patients and thus maintaining the benefits of completing concurrent chemoradiation. Notably, there were no substantial differences in completion of concurrent therapy in this trial, although induction TPF was still associated with high rates of toxicity. Similar to DeCIDE, the PARADIGM trial was underpowered due to slow enrollment and lower than expected event rates. There were no significant differences between the arms for survival outcomes. While the distant metastasis rate was lower in the induction arm (7 vs 11\%), this was offset by a higher rate of locoregional failures (13\% vs $8 \%)$, ultimately resulting in no difference in total failure rate between the arms.

Together, these three major Phase III trials (Hitt et al., DeCIDE, and PARADIGM) were not able to establish a survival advantage for TPF induction over standard cisplatin-based concurrent chemoradiation. This was largely explained by treatment-limiting toxicity, or possibly by the use of carboplatin rather than cisplatin in the concurrent component of therapy, such that improvements in distant control were negated by higher rates of locoregional failure. Since radiation therapy is the definitive treatment that drives locoregional control, administration of agents that interfere with radiation compliance and completion are difficult to justify in

\author{
“Arguments were made \\ that correct exploitation of \\ induction chemotherapy \\ might reduce distant \\ failures ... and thus increase \\ survival."
}


“...induction chemotherapy may play a role in de-intensification of treatment, using induction as a tool to stratify patients by treatment response." routine practice in the absence of a clear survival benefit. However, important shortcomings in these studies were noted. First, both DeCIDE and PARADIGM were underpowered for their primary end point. This was partially explained by lower-than-expected event rates, perhaps due to the rising incidence of human papillomavirus (HPV)-associated oropharyngeal cancers (OPC) and their predominance in the study populations. HPV-associated OPC is genetically distinct from cancers traditionally associated with tobacco and alcohol and carries a much better prognosis [21]. The importance of HPV as a prognostic factor was not well appreciated when these trials were designed, and patients were not stratified by this biomarker. Second, only DeCIDE limited enrollment to patients at high risk for distant metastasis. A trend for survival benefit was indeed seen in a high-risk subset of this study, but was limited by lack of power.

\section{Induction to select for reduced-intensity radiation-based treatment}

While these trials appeared to mark the end of induction, the arrival of EGFR inhibitors revived interest in the induction strategy. EGFR is highly expressed on head and neck squamous cell carcinomas and is quantitatively associated with worse outcomes [22]. In 2006, the US FDA approved the monoclonal EGFR antibody cetuximab for head and neck cancers, based on a randomized trial showing a 20 -month median survival advantage in favor of concurrent cetuximab versus radiation alone [23]. Furthermore, $94 \%$ of patients in that trial completed all cycles of cetuximab, a much higher completion rate than typically seen with concurrent cisplatin. Although the subsequent multi-institutional trial RTOG 0522 found no advantage in adding cetuximab to chemoradiation with cisplatin [24], the demonstrated effect and tolerability of the drug seemed to hold promise for the management of head and neck cancer.

One potential use was illustrated in the French TREMPLIN trial for laryngeal cancer [25]. Patients received TPF induction chemotherapy, and then were randomized to radiation with concurrent cisplatin or cetuximab. The implication was that cetuximab might improve the overall tolerability and completion rate of the chemoradiation phase of treatment and perhaps an advantage in survival might emerge. However, this trial found no differences in larynx preservation, laryngoesophageal dysfunction-free survival, or overall survival between the two arms, albeit with limited power to exclude any differences. The Spanish Head and Neck Cancer Group recently presented preliminary results from their trial 2007-01, which expanded this same concept to other sites [26]. Patients with unresectable disease ( $43 \%$ of whom had OPC) received TPF induction and were then randomized to cisplatin or cetuximab concurrent to radiation. Designed as a noninferiority trial, this study also found no difference in response rates or locoregional control between arms, although survival results are pending. While neither TREMPLIN nor this study included a standard of care comparator, they do offer the useful knowledge that if patients begin treatment with TPF induction (e.g., due to excessive delays beginning radiotherapy), they may receive concurrent cetuximab-radiation if they are unable to tolerate additional cisplatin, without fear of significantly compromising outcomes.

The French GORTEC group also recently presented early results of their trial 2007-02 [27]. Like the DeCIDE study, this trial limited enrollment to patients at high risk for distant metastasis, who in this case had nodal stage N2b or higher (with over $65 \%$ of participants having at least stage N2c). The control arm received radiotherapy with concurrent carboplatin/5FU, a standard European regimen. The experimental arm received induction TPF followed by concurrent cetuximab and radiation. After 32 months of median follow-up, there were no differences in progression-free survival, overall survival, or locoregional control. Provocatively, and reminiscent of the DeCIDE results, distant metastasisfree survival was improved and distant metastasis as a first event was significantly reduced in the induction arm. However, acute toxicity with induction was also higher, most strikingly with $17 \%$ of patients developing febrile neutropenia and $7 \%$ dying during induction chemotherapy. Detailed toxicity results are yet to be published, but these treatment-related mortality rates were substantially higher than the $0-3 \%$ seen in other trials using induction TPF $[19,20]$. This trial reiterated that induction TPF followed by concurrent cetuximab-radiation did not produce any survival benefit, and there were persistent concerns regarding toxicity.

\section{Is there a future for induction chemotherapy?}

Current directions of inquiry for induction chemotherapy may be broadly grouped into 
intensification and de-intensification. For intensification, results from the DeCIDE and GORTEC 2007-02 trials suggest that there may still be patients at the very highest risk for metastasis who could benefit from the addition of induction chemotherapy. However, they also indicate that a higher nodal stage, at least according to current staging criteria, may not be sufficient on its own to identify this group. Features such as low neck nodes and matted nodes (a proxy for extranodal extension of disease) are not clearly demarcated by the present American Joint Committee on Cancer staging system but may be more predictive of distant metastatic risk [28]. Protein expression biomarkers of aggressive disease may also identify patients who could benefit from induction taxane chemotherapy [29-31]. Another area open to exploration is oligometastatic disease. Roughly $15 \%$ of head and neck cancer patients will present with distant metastases, but a portion of these will have only a few discrete lesions. The increasing sophistication and clinical experience of stereotactic body radiotherapy has made definitive local treatment to these sites a reasonable option [32]. Strategies may emerge combining induction chemotherapy and upfront metastasis-directed treatments prior to locoregional therapy for the primary tumor.

Conversely, induction chemotherapy may play a role in de-intensification of treatment, using induction as a tool to stratify patients by treatment response. This could be especially applicable to good-prognosis HPV-associated OPC. There are two American trials testing induction chemotherapy in this manner which have not yet been completed, OPTIMA HPV (NCT02258659) and the Quarterback Trial (NCT01706939), and one which has just been reported, Eastern Cooperative Oncology Group (ECOG) 1308 (NCT01084083). The ECOG 1308 trial was a single-arm Phase II study in which patients with HPV-associated OPC received induction paclitaxel/cisplatin/cetuximab and cetuximab concurrently with radiation [33]. The radiation was dose-reduced from 69.3 to 54 Gy to the primary site if there was a clinical complete response to induction; involved lymph nodes received 69.3 Gy unless they also were judged to have completely responded. 56 of 80 evaluable patients (70\%) achieved a primarysite clinical complete response after induction and 51 patients continued to cetuximab with 54 Gy. At a median follow-up of 35.4 months, the 2-year progression-free survival and overall survival rates were $80 \%$ and $94 \%$ among those 51 patients; among patients who received a standard dose of radiation, the rates were $67 \%$ and $87 \%$, respectively. The poor outcome for nonresponders may have been confounded by clinical characteristics, which were seemingly as predictive as the response to induction: 2-year progression-free survival was $96 \%$ for patients with a history of ten or less pack-years of smoking and less than T4 and N2c stage, versus a rate of $71 \%$ for those with T4 or N2c stage or more than ten pack-years of smoking. This is consistent with the multiple recursive-partitioning analysis of RTOG 0129 [21], and it emphasizes that patient selection requires further refinement in order to realize benefits from induction.

\section{Conclusion}

As of 2016 induction chemotherapy has no clearly established frontline role in the routine treatment of head and neck carcinomas of the major non-nasopharyngeal sites. Platinum-based concurrent chemoradiation remains the standard option, with the possible exceptions of organ preservation for laryngeal and hypopharyngeal cancers, where induction is at most a reasonable alternative. Evidence from multiple randomized controlled studies indicates that induction before concurrent chemoradiation is not substantially inferior in efficacy, although it is generally more toxic and costly. Thus, outside of a clinical trial the utility of induction chemotherapy is restricted to uniquely pragmatic clinical scenarios where radiation is unavoidably delayed or cannot be tolerated. Some examples are severe pain from advanced disease that renders the radiation setup unstable, or impending airway compromise or neurological dysfunction that necessitates rapid initiation of treatment. EGFR-targeted antibodies are potential substitutes for cisplatin in patients who have completed induction and are proceeding to concurrent chemoradiation. Future areas of study may include intensification of therapy for very high-risk or oligometastatic patients, but selection criteria are not yet optimized. Induction as a strategy for subsequent response-based deintensification is being explored, especially in HPV-associated disease. However, selection of the ideal candidates for this approach likewise remains undefined. Future novel approaches will likely feature radiographic, proteomic and genomic biomarkers used to define prognostic groups and guide treatment selection with greater precision. Because of its flexibility and “...outside of a clinical trial the utility of induction chemotherapy is restricted to uniquely pragmatic clinical scenarios where radiation is unavoidably delayed or cannot be tolerated.” 
adaptability, induction will likely continue to be a 'testing site' for new chemotherapeutic, targeted or immunologic agents.

\section{Financial \& competing interests disclosure}

SS Yom received a research grant from Genentech, honorarium from Astra-Zeneca, and royalty from UpToDate.

The authors have no other relevant affiliations or financial involvement with any organization or entity with a financial interest in or financial conflict with the subject matter or materials discussed in the manuscript apart from those disclosed.

No writing assistance was utilized in the production of this manuscript.

\section{Open access}

This work is licensed under the Creative Commons Attribution-NonCommercial 4.0 Unported License. To view a copy of this license, visit http://creativecommons.org/ licenses/by-nc-nd/4.0/

\section{References}

1 Chapman CH, Parvathaneni U, Yom SS. Revisiting induction chemotherapy before radiotherapy for head and neck cancer, part II: nasopharyngeal carcinoma. Future Oncol. 13(7), 581-584 (2017)

2 Pignon JP, Bourhis J, Domenge C, Designé L. Chemotherapy added to locoregional treatment for head and neck squamous-cell carcinoma: three meta-analyses of updated individual data. Lancet 355(9208), 949-955 (2000).

3 Pignon J-P, le Maître A, Maillard E, Bourhis J; MACH-NC Collaborative Group.

Meta-analysis of chemotherapy in head and neck cancer (MACH-NC): an update on 93 randomised trials and 17,346 patients. Radiother. Oncol. 92(1), 4-14 (2009).

4 Denis F, Garaud P, Bardet E et al. Final results of the 94-01 French Head and Neck Oncology and Radiotherapy Group randomized trial comparing radiotherapy alone with concomitant radiochemotherapy in advanced-stage oropharynx carcinoma. J. Clin. Oncol. 22(1), 69-76 (2004).

5 Adelstein DJ, Li Y, Adams GL et al. An intergroup Phase III comparison of standard radiation therapy and two schedules of concurrent chemoradiotherapy in patients with unresectable squamous cell head and neck cancer. J. Clin. Oncol. 21(1), 92-98 (2003).

6 Wolf GT, Hong WK, Fisher SG et al. Induction chemotherapy plus radiation compared with surgery plus radiation in patients with advanced laryngeal cancer. The Department of Veterans Affairs Laryngeal Cancer Study Group. N. Engl. J. Med. 324(24), 1685-1690 (1991).

7 Lefebvre JL, Chevalier D, Luboinski B, Kirkpatrick A, Collette L, Sahmoud T. Larynx preservation in pyriform sinus cancer: preliminary results of a European Organization for Research and Treatment of Cancer Phase III trial. EORTC Head and
Neck Cancer Cooperative Group. J. Natl Cancer Inst. 88(13), 890-899 (1996).

8 Lefebvre JL, Rolland F, Tesselaar M et al. Phase III randomized trial on larynx preservation comparing sequential vs alternating chemotherapy and radiotherapy. J. Natl Cancer Inst. 101(3), 142-152 (2009).

9 Weber RS, Berkey BA, Forastiere A et al. Outcome of salvage total laryngectomy following organ preservation therapy: the Radiation Therapy Oncology Group trial 91-11. Arch. Otolaryngol. Head Neck Surg. 129(1), 44-49 (2003).

10 Forastiere AA, Zhang Q, Weber RS et al. Long-term results of RTOG 91-11: a comparison of three nonsurgical treatment strategies to preserve the larynx in patients with locally advanced larynx cancer. J. Clin. Oncol. 31(7), 845-852 (2013).

11 Forastiere AA, Neuberg D, Taylor SG, DeConti R, Adams G. Phase II evaluation of taxol in advanced head and neck cancer: an Eastern Cooperative Oncology Group trial. J. Natl Cancer Inst. Monogr. (15), 181-184 (1993).

12 Hitt R, López-Pousa A, Martínez-Trufero J et al. Phase III study comparing cisplatin plus fluorouracil to paclitaxel, cisplatin, and fluorouracil induction chemotherapy followed by chemoradiotherapy in locally advanced head and neck cancer. J. Clin. Oncol. 23(34), 8636-8645 (2005).

13 Vermorken JB, Remenar E, van Herpen C et al. Cisplatin, fluorouracil, and docetaxel in unresectable head and neck cancer. $N$. Engl. J. Med. 357(17), 1695-1704 (2007).

14 Posner MR, Hershock DM, Blajman CR et al. Cisplatin and fluorouracil alone or with docetaxel in head and neck cancer. $N$. Engl. J. Med. 357(17), 1705-1715 (2007).

15 Pointreau Y, Garaud P, Chapet $S$ et al. Randomized trial of induction chemotherapy with cisplatin and 5-fluorouracil with or without docetaxel for larynx preservation. J. Natl Cancer Inst. 101(7), 498-506 (2009).
16 Janoray G, Pointreau Y, Garaud P et al. Long-term results of a multicenter randomized Phase III trial of induction chemotherapy with cisplatin, 5-fluorouracil, \pm docetaxel for larynx preservation. J. Natl Cancer Inst. 108(4), djv368 (2016).

17 Brockstein B, Haraf DJ, Rademaker AW et al. Patterns of failure, prognostic factors and survival in locoregionally advanced head and neck cancer treated with concomitant chemoradiotherapy: a 9-year, 337-patient, multi-institutional experience. Ann. Oncol. 15(8), 1179-1186 (2004).

18 Hitt R, Grau JJ, Lopez-Pousa A et al. A randomized Phase III trial comparing induction chemotherapy followed by chemoradiotherapy versus chemoradiotherapy alone as treatment of unresectable head and neck cancer. Ann. Oncol. 25(1), 216-225 (2014).

19 Cohen EEW, Karrison TG, Kocherginsky M et al. Phase III randomized trial of induction chemotherapy in patients with $\mathrm{N} 2$ or N3 locally advanced head and neck cancer. J. Clin. Oncol. 32(25), 2735-2743 (2014).

20 Haddad R, O'Neill A, Rabinowits G et al. Induction chemotherapy followed by concurrent chemoradiotherapy (sequential chemoradiotherapy) versus concurrent chemoradiotherapy alone in locally advanced head and neck cancer (PARADIGM): a randomised Phase III trial. Lancet Oncol. 14(3), 257-264 (2013).

21 Ang KK, Harris J, Wheeler R et al. Human papillomavirus and survival of patients with oropharyngeal cancer. N. Engl. J. Med. 363(1), 24-35 (2010).

22 Ang KK, Berkey BA, Tu X et al. Impact of epidermal growth factor receptor expression on survival and pattern of relapse in patients with advanced head and neck carcinoma. Cancer Res. 62(24), 7350-7356 (2002).

23 Bonner JA, Harari PM, Giralt J et al. Radiotherapy plus cetuximab for locoregionally advanced head and neck 
cancer: 5-year survival data from a Phase III randomised trial, and relation between cetuximab-induced rash and survival. Lancet Oncol. 11(1), 21-28 (2010).

24 Ang KK, Zhang Q, Rosenthal DI et al. Randomized Phase III trial of concurrent accelerated radiation plus cisplatin with or without cetuximab for stage III to IV head and neck carcinoma: RTOG 0522. J. Clin. Oncol. 32(27), 2940-2950 (2014).

25 Lefebvre JL, Pointreau Y, Rolland F et al. Induction chemotherapy followed by either chemoradiotherapy or bioradiotherapy for larynx preservation: the TREMPLIN randomized Phase II study. J. Clin. Oncol. 31(7), 853-859 (2013).

26 Hitt R, Mesia R, Grau JJ et al. Randomized Phase III trial of induction chemotherapy (ICT) with docetaxel-cisplatin-5fluorouracil (DCF) followed by cisplatin-radiotherapy (CRT) or cetuximab-radiotherapy (CetRT) in patients (pts) with locally advanced unresectable head and neck cancer
(LAUHNC). J. Clin. Oncol. 34(Suppl.), Abstract 6001 (2016).

27 Geoffrois L, Martin L, Garaud P et al. Induction docetaxel platinum 5-FU (TPF) followed by cetuximab-radiotherapy (cetux-RT) versus concurrent chemoradiotherapy $(\mathrm{CT} / \mathrm{RT})$ in patients with $\mathrm{N} 2 \mathrm{~b} / \mathrm{c}-\mathrm{N} 3$ non operated stage III-IV squamous cell cancer of the head and neck (SCCHN): results of the GORTEC 2007-2002 Phase III randomized trial. J. Clin. Oncol. 34(Suppl.), Abstract 6000 (2016).

$28 \mathrm{Kim}$ DH, Kim WT, Lee JH et al. Analysis of the prognostic factors for distant metastasis after induction chemotherapy followed by concurrent chemoradiotherapy for head and neck cancer. Cancer Res. Treat. 47(1), 46-54 (2014).

29 Zhu D-W, Liu Y, Yang X et al. Low annexin A1 expression predicts benefit from induction chemotherapy in oral cancer patients with moderate or poor pathologic differentiation grade. BMC Cancer 13(1), 301 (2013).
30 Saba NF, Magliocca KR, Kim S et al. Acetylated tubulin (AT) as a prognostic marker in squamous cell carcinoma of the head and neck. Head Neck Pathol. 8(1), 66-72 (2014).

31 Yang CZ, Ma J, Zhu DW et al. GDF15 is a potential predictive biomarker for TPF induction chemotherapy and promotes tumorigenesis and progression in oral squamous cell carcinoma. Ann. Oncol. 25(6), 1215-1222 (2014).

32 Florescu C, Thariat J. Local ablative treatments of oligometastases from head and neck carcinomas. Crit. Rev. Oncol. Hematol. 91(1), 47-63 (2014).

33 Marur S, Li S, Cmelak AJ et al. E1308: Phase II trial of induction chemotherapy followed by reduced-dose radiation and weekly cetuximab in patients with HPV-associated resectable squamous cell carcinoma of the oropharynx ECOG-ACRIN Cancer Research Group. J. Clin. Oncol. doi: 10.1200/JCO.2016.68.3300 (2016) (Epub ahead of print). 